

\section{La enseñanza de la geografía desde una perspectiva socioespacial. Caso de enfoque en una asistencia técnica con docentes de Chile, Argentina y Colombia}

Teaching Geography from a Socio-spatial Perspective. Case of a Teacher Training Process in Chile, Argentina and Colombia

Ensino de Geografia desde uma perspectiva sócio-espacial. Caso do num processo de formação docente no Chile, na Argentina e na Colômbia

Alejandro Pimienta Betancur*

Raquel Pulgarín Silva**

Ana Scoones Landoni ${ }^{* * *}$

Andoni Arenas Martija ${ }^{* * * *}$

Rosser Bianchi Parraguez ${ }^{* * * *}$

\footnotetext{
Profesor Universidad de Antioquia, Colombia.

** Profesora Universidad de Antioquia, Colombia.

*** Profesora Universidad Nacional de Cuyo, Argentina.

**** Profesor Pontificia Universidad Católica de Valparaíso, Chile.

***** Profesor Universidad Católica Silva Henríquez, Chile.
} 


\section{Resumen}

El texto presenta la experiencia de formulación y diseño de un proyecto de actualización docente sobre educación en riesgos socioambientales, realizado con docentes de cuatro ciudades latinoamericanas: Santiago y Valparaíso (Chile), Mendoza (Argentina) y Medellín (Colombia). Dicho proceso permitió al grupo de profesores orientadores reflexionar su propio quehacer desde un ejercicio colectivo, trascendiendo la particularidad del curso y de los casos locales, para plantear un posicionamiento sobre la formación continua y la actualización de los docentes en América Latina. Se problematiza la tendencia de restringir

\section{Abstract}

This article presents the designing experience of a project for teachers updating on socioenvironmental risk education, carried out with teachers from four Latin American cities: Santiago and Valparaíso (Chile), Mendoza (Argentina) and Medellín (Colombia). That process allowed the group of guiding teachers to reflect on their own work from a collective exercise. Transcending the particularity of the courses and local cases to propose a position on continuous formation and upgrading of teachers in Latin America. It is problematized the tendency to restrict didac-

\section{Resumo}

O texto oferece a experiência de formulação e desenho de um projeto de atualização docente sobre a educação em riscos socioambientais, realizado com docentes de quatro cidades latino-americanas: Santiago e Valparaíso (Chile), Mendoza (Argentina) e Medellín (Colômbia). Desse processo permitiu ao grupo de professores orientadores, refletir seu próprio fazer desde um exercício coletivo, transcendendo a particularidade do curso e dos casos locais, para planejar um posicionamento coletivo, sobre a formação contínua e a atualização dos docentes na América Latina. Se problematiza uma tendência de restringir a didática la didáctica a las denominadas innovaciones que permiten enseñar más rápido, y se plantea un enfoque elaborado para la didáctica de la geografía que dialogue con la educación geográfica. A partir de ello, se desarrolla una estrategia en la que cobra valor comparar casos locales como posibilidad de problematizar lo particular y lo universal en pro de renovar la enseñanza de la geografía.

\section{Palabras clave}

didáctica de la geografía, formación docente, riesgos socioambientales, educación geográfica.

tics to the so-called innovations that allow to teach faster, and consequently an elaborated approach is proposed for the geography didactics that dialogue with the geographical education. From the prior a strategy is developed where is worth it to compare local cases, as a possibility to problematize the particular and the universal, in order to renew the teaching of geography.

\section{Keywords}

Didactics of geography, teacher formation, socioenvironmental risks, geographic education. as denominadas inovações que permitem ensinar mais rápido e, planejar um enfoque elaborado para a didática da geografia que dialogue com a educação geográfica. A partir da qual se desenvolva uma estratégia donde seja cobrado valor ao comparar casos locais como possibilidade de problematizar o particular e o universal, em prol de revonar o ensino de geografia.

\section{Palavras clave}

didática da geografia, formação docente, riscos socioambientais, educação geográfica. 


\section{Introducción}

A partir de la experiencia de formulación y diseño del curso B-learning sobre educación en riesgos socioambientales, dirigido a 40 docentes de cuatro ciudades latinoamericanas: Santiago y Valparaíso (Chile), Mendoza (Argentina) y Medellín (Colombia), y que se desarrolla entre julio y noviembre del 2016, el grupo de profesores orientadores presenta la siguiente reflexión sobre su propio ejercicio colectivo, buscando trascender la particularidad del curso y de los casos locales, para plantear un posicionamiento colectivo para la formación continua y actualización de los docentes en América Latina.

Este texto funge como lugar de enunciación para la acción y sirve de antesala para el desarrollo de un proyecto de asistencia técnica denominado "Incentivo para el conocimiento geográfico y la resolución de problemas espaciales destinado a profesores de enseñanza básica/primaria en países latinoamericanos". El equipo responsable del proyecto, los autores de este artículo, parten de la idea de que la formación del educador de geografía es un proceso constante cuyo fin es conseguir en el estudiante el aprendizaje de la ciencia, el conocimiento de los problemas ambientales, para este caso, y el cuidado y el respeto por los riesgos. En particular, este curso, que se desarrolla en la modalidad B-learning (mixta virtual-presencial), busca que los docentes se cualifiquen para implementar procesos de enseñanza-aprendizaje relevantes en torno a los temas de desastres naturales/humanos y cambio climático, acorde a la realidad de distintos países. Entonces, la pregunta clave es ¿cómo evitar la didáctica restringida y quedarse en la particularidad del cómo, y avanzar para configurar este espacio en un proceso de didáctica elaborada, es decir, una formación en didáctica de la geografía que piense la triada cómo, para qué y por qué?

Este ejercicio se inscribe en la lógica de la investigación sobre la práctica, en este caso de formación continua de docentes, que sirve de antesala a un ejercicio sistemático de investigación comparada en este campo, en el cual se analizará la ejecución del proceso. Por tanto, se puede considerar un avance reflexivo en el que el grupo de profesores orientadores propone alternativas a la tendencia hegemónica de restringir la didáctica a las denominadas innovaciones que permitan enseñar más rápido, y se posiciona en un enfoque elaborado para la didáctica de la geografía y lo relaciona con la educación geográfica, lo cual se expone en la primera parte del escrito. Después, en la segunda y tercera parte del texto, se muestra la relación entre lo particular y lo universal, y se puntualizan los casos. Finalmente, a modo de conclusión, se abren unas preguntas para darle continuidad al ejercicio investigativo de comparación.

\section{Lugar de enunciación: hacia la didáctica de la geografía con código elaborado}

La didáctica de la geografía se configura en la actualidad como un discurso cuyo sentido educativo es disputado por los cambios recientes promovidos por las reformas pedagógicas en el continente, que vienen configurando un currículo hegemónico. En esa construcción de sentido hegemónico, a la didáctica en general y, por efecto de cascada, a la didáctica de las ciencias sociales y de la geografía las sigue eclipsando la significación que las circunscribe como un procedimiento de aplicación, solamente ligado a la pregunta de cómo enseñar, lo cual es la versión restringida de la didáctica, sobre la versión antagónica que formulan muchos docentes e investigadores especializados en este campo que reclaman una y otra vez que de ninguna manera se puede pensar el cómo, sin el qué y el para qué, es decir, una versión elaborada de la didáctica, la cual suscribimos. Esta versión elaborada de la didáctica es la que dialoga con la educación geográfica con una pretensión de no quedarse en un particular sino entender la totalidad.

La didáctica de la geografía, si bien se ha venido constituyendo como un campo de conocimiento y de formación de docentes, aún no es un discurso muy consolidado ni con mucha especificidad respecto a campos más amplios como el de la didáctica de las ciencias sociales, la enseñanza de la geografía y la educación geográfica. Aunque para muchos esta suerte de indeterminación es un asunto de discusión intelectual entre universitarios mediante productos académicos (libros, artículos, etc.) para determinar campos y objetos, la realidad es que al nivel de las políticas educativas ya hay mucho avance en intervenir el ejercicio de la docencia desde la perspectiva de la didáctica restringida. Es decir, mientras los investigadores se debaten los límites y las colindancias de la didáctica de la geografía, el currículo hegemónico viene ajustando los clivajes estratégicos para que la didáctica restringida sea el campo de acción del docente. Lo que está en juego es la estructuración general de la geografía escolar, su construcción de sentido y el lugar que tiene el conocimiento geográfico en la formación de los ciudadanos.

Aquello que se debe problematizar para entender el debate entre la didáctica restringida y la didáctica elaborada de la geografía, y sus relaciones con los discursos de la enseñanza de la geografía y la educación geográfica, tiene que ver con el uso político del discurso de la didáctica. Veamos: la mayoría de reformas educativas de los últimos veinte años en América Latina y las políticas educativas que rigen el currículo explícito, si bien se hacen en nombre del mejoramiento de la calidad educativa, enuncian visiones sistémicas, integrales y holísticas, señalan la centralidad del papel del docente en el aprendizaje, el cual debe ser pertinente, entendido como un saber hacer (habilidades y destrezas) para vivir en una sociedad global, ha ido produciendo otros efectos no explícitos. Para el caso, lo que interesa señalar es que la especificidad de la geografía 
escolary de las disciplinas escolares se ha debilitado porque ha perdido importancia relativa dentro del currículo, y ha dado lugar a enfoques y perspectivas integradoras y transversales que buscan un aprendizaje tipo competencias.

Se reconoce que hay argumentos muy autorizados que esgrimen que dicho debilitamiento no implica que se haya disminuido el aprendizaje de lo social y, al contrario, argumentan que tanto el cambio de la enseñanza de las disciplinas, que muchas veces terminaba enfocándose en contenidos abstractos, como la implementación de una enseñanza para el aprendizaje de competencias basada en ámbitos conceptuales interdisciplinarios y relacionada con el análisis de problemas sociales y del medio, han permitido un incremento en el valor social de las ciencias sociales. Es decir, para algunos analistas y tomadores de decisiones no hay correlación entre el debilitamiento de la geografía escolar como área específica del currículo y la enseñanza o la educación geográfica. Simplemente han cambiado las formas curriculares y se han adaptado a las condiciones de la sociedad contemporánea, pero en la escuela sigue vigente la educación geográfica. Otros, por el contrario, entienden que sí existe tal correlación y la educación geográfica se ha debilitado a la par de la geografía escolar como área específica.

Teniendo en cuenta el debate de esa correlación entre área o asignatura de geografía escolar y enseñanza o educación geográfica en las recientes reformas educativas, lo que interesa problematizar en esta ponencia es una arista más concreta de ese problema. Por efecto de dichas reformas y la configuración de lo que se podría denominar el discurso del currículo hegemónico para América Latina, se ha aumentado la brecha entre los que toman las decisiones sobre el currículo explícito, por ejemplo, los que tomaron la decisión de debilitar la geografía escolar como área bajo el supuesto de fortalecer la educación geográfica, y los que la enseñan en las escuelas, es decir, los docentes de oficio. Y en ese currículo hegemónico, la ampliación de esa brecha ubica a cada actor en un juego de posiciones, una suerte de distribución del trabajo educativo, en la cual unos piensan el currículo y entienden su totalidad, y otros lo aplican desde la particularidad.

En ese sentido, el efecto es que el docente que enseña geografía tiene una acción cada vez más particular y no se ocupa de pensar el qué y el para qué enseña, es decir, la totalidad. El discurso del currículo hegemónico le da centralidad al maestro en esa particularidad. Podríamos decir que, volviendo a lo que dijimos al principio, al docente solo se le está pidiendo que se especialice en la didáctica del código restringido: que aplique, que transmita. No se le pide que piense en términos de la didáctica elaborada, la cual es cercana a los discursos pedagógicos de la enseñanza y la educación geográfica. Es preciso, desde una perspectiva política y pedagógica, disputar el sentido de la didáctica en función de su versión elaborada, nunca como una aplicación, tal como viene consolidándose en el currículo hegemónico. Se ve, entonces, que no es un asunto semántico ni de intelectuales, es una disputa por el sentido de lo que hacemos.

Si asumimos esa dicotomía que establece que la educación geográfica es el campo para pensar y la didáctica de la geografía es el campo del hacer, estaremos dejando que todo tipo de programas de asistencia técnica, de actualización docente, incluso la formación inicial de maestros tienda cada vez más a esa didáctica restringida, y la reflexión se quede en las universidades y en los centros de investigación, es decir, ampliando aún más las brechas.

\section{Enfoque: el valor de comparar o la necesidad superar la impaciencia}

La estrategia para tal fin es que el curso permita la reflexión constante en términos didácticos entre la particularidad que hay entre cada caso (problema socioespacial por ciudad: Medellín, Valparaíso, Mendoza y Santiago) y lo universal de la escala latinoamericana y global. Es decir, se aprovecha el valor de comparar lo diverso de manera que el docente no se concrete en entender una secuencia didáctica para aplicar en un caso, sino que se aproveche la diversidad de participantes y experiencias que confluyen en el curso, de manera que se genere una interlocución que se salga de la restricción que impone el currículo hegemónico, es decir, que el docente supere la impaciencia de resolver el cómo y se concentre en entender el proceso.

Nos referimos a la necesidad de superar la impaciencia, inspirados en Zigmund Bauman (2007) cuando, en Los retos de la educación en la modernidad líquida, reflexiona sobre el significado del tiempo en nuestros días y cómo toda demora, dilación o espera se ha transformado en un estigma de inferioridad. Quienes deben esperar pertenecen a una jerarquía inferior. Al contrario, para los que están en la jerarquía superior, el tiempo es el emblema de privilegio, que les permite "el acceso a los atajos, a los medios que permiten alcanzar la gratificación instantáneamente” (p. 22). Bauman (2007) llama a esa situación el síndrome de la impaciencia.

Muchas de las últimas reformas que se han introducido de manera exógena a la educación se explican por el afán de adaptarla a dicho síndrome, en tanto el valor de lo que se aprende ya no está en el proceso de aprenderlo, es decir, ya no radica en el proceso sino en el producto. Como producto "la educación ha pasado a ser una cosa que se consigue, completa y terminada, o relativamente acabada" (Bauman, 2007, p. 24), lo cual no es difícil de comprobar en las diferentes experiencias nacionales en América Latina.

Muchos docentes se han posicionado en esta perspectiva y creen que su función es enseñar rápido lo más útil, para lo cual adoptan innovaciones didácticas y se capacitan, lo que les calma el síndrome de la impaciencia 
y, por supuesto, también a los directivos, políticos, padres de familia, estudiantes, etc. La dificultad es cuando esto no va acompañado del pensar, ejercicio que implica salir del particular que restringe y se eleva hacia el universal.

Este círculo de presión sobre la didáctica para que sea solo una didáctica restringida es ejercido desde el currículo hegemónico, entendido como el discurso educativo que subyace a la hegemonía política contemporánea y que trata de ordenar, desde el ejercicio de la predeterminación, toda la educación, incluyendo sus actores, sus acciones, sus procesos y sus resultados. Es decir, las innovaciones didácticas que se promueven desde el currículo hegemónico no pueden entenderse como buenas intenciones que permiten intervenciones concretas para enseñar mejor, sino como componentes de un proceso integral de transformación de la educación para que sea consistente con la hegemonía política. Así, el síndrome de la impaciencia del cual habla Bauman es una expresión de la hegemonía política contemporánea.

En términos de la formación docente, como es el caso del curso de referencia, lo que permite superar el síndrome de la impaciencia es lograr un buen equilibrio entre lo particular de cada caso que se va a analizar y lo universal de la reflexión de la educación geográfica, es decir, el valor de comparar.

\section{Los casos: de lo particular a lo universal de las preguntas cómo, por qué y para qué}

La capacitación y la actualización de los docentes de geografía y ciencias sociales en América Latina no pueden limitarse a contextualizar secuencias didácticas innovadoras en los currículos oficiales, como si la realidad fuera el currículo. Por supuesto, el currículo es un elemento ordenador de la práctica docente y no puede ser despreciado, pero no es el único elemento. Así, en el curso se reconoce la importancia del currículo, en este caso los currículos nacionales y locales, pero se le da prioridad a entender la realidad social desde una perspectiva socioespacial, es decir, entender que las manifestaciones sociales y naturales que rodean al estudiante para el caso, asociadas con riesgos, pueden ser entendidas desde coordenadas espacio-temporales, de manera que se comprendan mejor las causas y las consecuencias de una manera integral, no solo desde los conceptos.

Los casos que se abordan en el curso son cuestiones socialmente vivas, es decir, fenómenos relevantes y significativos para las personas que lo viven y sienten (desde la experiencia espacial) y se abordan de manera compleja como problemas socioespaciales, evitando tratarlos desde meros conceptos. En todos los casos, su abordaje socioespacial permite entender (y enseñar) la configuración sociohistórica del ser humano con el medio (perspectiva constitutiva del espacio), y los múltiples relacionamientos escalares y tensiones local-global (perspectiva relacional), lo que posibilita tomar decisiones didácticas de cuáles conceptos y cuáles habilidades se van a tratar, pero sin perder de vista la universalidad del por qué y el para qué.

Por tanto, el enfoque del curso parte de las cuestiones socialmente vivas o significativas para el estudiante por el riesgo que puede implicar y las aborda como problemas socioespaciales. Además, la diversidad de casos es el mayor potencial porque permite que los docentes no se queden enfrascados en la particularidad de un caso y de un currículo, sino que pueden ver y comparar otros casos y formas de contextualizarlos en los currículos. Con esa estrategia se busca que el docente pueda acceder tanto a lo particular como a lo universal, teniendo en mente no solo el cómo, sino también el por qué y el para qué. Así, se aporta a que el docente tenga mejores posibilidades de tener una didáctica elaborada, en la que la enseñanza de la geografía sea un proceso intencionado y complejo que le permite entender el currículo sin volverlo esencia, como una metarrealidad, y darle de esa manera un sentido a su finalidad: la educación geográfica.

A continuación se aportan los elementos contextuales de los currículos nacionales y los casos específicos que se han elegido para este curso: en Santiago el caso local es "problemas y desafíos de la cuenca de Santiago"; en Valparaíso son "tsunamis producidos por terremotos"; en Mendoza es "la prevención sísmica" y en Medellín "la contaminación atmosférica”.

\section{El caso de Argentina}

La estructura curricular implementada con la Ley de Educación Nacional (Ley 26.206 del 2006) incluye la geografía en el área de las ciencias sociales, para la escuela primaria y secundaria. Tanto los diseños curriculares de las provincias (jurisdicción menor) como de la Nación expresan una concepción del espacio geográfico entendida como el resultado de una construcción social, que incorpora los procesos históricos y las distintas escalas de análisis para la comprensión de las configuraciones espaciales. La perspectiva ambiental, según los documentos oficiales, integra los elementos físico-naturales y los componentes de tipo social. En cuanto a las metodologías de enseñanza, proponen una mirada compleja y totalizadora de la realidad social, integrando distintas dimensiones en las explicaciones de los fenómenos geográficos.

Uno de los ejes fundamentales en el estudio de la dimensión físico-natural son los procesos que han dado lugar a la formación de los distintos ambientes. Los riesgos y los desastres naturales no aparecen en el currículo escolar. Su estudio, en algunos casos, aparece en el área de las ciencias naturales (tales como los fenómenos geológicos), constituyendo un contenido aislado del contexto del alumno. En el diseño correspondiente 
a la provincia de Mendoza, aparece entre los contenidos del área de las ciencias sociales como "algunos casos de riesgos y catástrofes ambientales en el planeta y su impacto en las sociedades" . La geografía del riesgo no está concretamente expresada en el currículo escolar, de allí que se deja librada a la decisión de las instituciones o los docentes abordar su enseñanza e incluir el tema como un contenido transversal.

Mendoza es una de las zonas de mayor riesgo sísmico en el territorio argentino. La ciudad capital, dada su proximidad con la cordillera de los Andes, está edificada sobre fallas activas y que han generado, y lo siguen haciendo, los movimientos sísmicos en la provincia. Históricamente, se recuerdan varios sismos con efectos destructivos. Si bien los registros certeros datan de la época colonial, existen relatos de otros fenómenos que provocaron desastres ambientales en tiempos pasados. El más conocido por los mendocinos es el terremoto de 1861 que destruyó la ciudad colonial y obligó a su reconstrucción en otro lugar diferente. El último sismo que provocó daños en los edificios y destrucción de casas de adobe se registró en 1985. El peligro de un desastre vive en la percepción que los habitantes de la provincia construyen sobre su ambiente. De allí que la capacidad de organización de la comunidad frente a estos eventos depende de un complejo sistema preventivo en el que intervienen distintos actores sociales. En este sentido, formar personas competentes a la hora de actuar con responsabilidad en determinadas situaciones de riesgo geológico es un compromiso que la educación no puede soslayar.

En Mendoza se realizan simulacros de sismos periódicos en las escuelas públicas y privadas, con participación de las instituciones involucradas, como Defensa Civil, bomberos, policía y la Cruz Roja. Sin embargo, se observa que esta práctica no se integra con un trabajo continuo y una propuesta curricular adecuada a los parámetros que se deben seguir para la gestión de riesgos.

La formación disciplinar desde la visión geográfica es un enfoque que contribuye a una concepción integradora del riesgo y a una percepción más clara de la necesidad de aplicar herramientas de planificación estratégica en el manejo del riesgo en el contexto de las políticas públicas sobre el territorio.

La enseñanza de la geografía, en general, en la escuela primaria se apoya en la descripción y la localización de los elementos constitutivos de la superficie terrestre, las características de la población, el aprovechamiento económico de los recursos naturales, entre otros aspectos. Esta acción pedagógica responde a las tradicionales formas de enseñar geografía, estructuradas en largas listas de preguntas y respuestas que obligan a la memorización. También aparece con frecuencia la actividad ligada al mapa como instrumento para la localización y la lectura de símbolos.

1 DCP. Diseño Curricular Provincial, gobierno de Mendoza, Cuaderno No39.
El desafío consiste en vincular los ámbitos instituciones, como el educativo, la ciencia y la técnica, el sector académico y los organismos oficiales encargados de la gestión del riesgo y la reducción de desastres. Tal como se ha expresado, la geografía del riesgo no es un contenido presente en el currículo escolar oficial, por tal motivo es que es vital la preparación del profesorado en conceptos, marcos teóricos, estrategias de enseñanza y de evaluación, apoyados en los avances científicos que, en el caso de Mendoza, se han alcanzado en cuanto a la actividad sísmica.

La educación en general juega un papel estratégico en la protección social, el desarrollo humano, y la sostenibilidad de las comunidades y los sistemas productivos. La educación sobre riesgos y desastres en América Latina no ha alcanzado el lugar que necesita para crear y construir uno de los principales pilares para una calidad de vida sostenible en toda comunidad: su propia seguridad frente a desastres naturales.

\section{El caso Chile}

Si bien las bases curriculares del 2012 de la asignatura de Historia, Geografía y Ciencias Sociales postulan como propósito de la enseñanza de la geografía escolar el desarrollo del pensamiento geográfico, la ausencia de densidad conceptual, de continuidad de contenidos, metas y temáticas geográficas, la insistencia en no establecer relaciones entre habilidades genéricas y aquellas que la geografía puede formar en el itinerario escolar, validan la aspiración y los fundamentos curriculares de un pensamiento geográfico escolar.

La disposición del currículo y su concreción en programas de los diferentes niveles/cursos no permiten ni facilitan la enseñanza de la geografía hacia el desarrollo escolar de un pensamiento geográfico. Desde este punto de vista, el currículo nacional de la geografía escolar adolece de una incoherencia esencial.

Temas nucleares que relevan la complejidad de "lo geográfico" desarrollados desde una didáctica elaborada de la geografía escolar, en los términos como ha sido explicada más arriba, parecen ser un modo sustantivo de al menos enfrentar esta incoherencia.

En el contexto del curso internacional sobre educación en riesgo que se hecho mención en esta ponencia, se ha optado por desarrollar dos temas nucleares de mesoescala en su ejemplificación y trabajar, que contienen en sí mismos la particularidad y la universalidad. Es decir, pueden tanto ser vivenciables-estudiables a la escala local por estudiantes y profesores, como también son la expresión de que se es parte de una realidad más amplia, de un todo de relaciones, que está más allá del espació vivencial: la cuenca metropolitana de Santiago y los tsunami producidos por terremotos que se desarrollan en la costa de Chile.

Es importante tener en cuenta en esta perspectiva que las condiciones ambientales-naturales de la cuenca de Santiago y de la ocurrencia de 
tsunamis solo explican en parte las situaciones de riesgo, puesto que la producción del espacio y las opciones de habitabilidad humana han transformado en gran medida esas condiciones.

Por ejemplo, en Santiago tanto el escurrimiento superficial de las aguas como la calidad del aire son esencialmente humanos; el litoral, la costa chilena, sobre todo en la zona central de Chile, han devenido en procesos de transformación urbana por el desarrollo inmobiliario de la segunda o tercera residencia, por ejemplo. Desde este enfoque, entonces, los riegos no son meramente adjudicables a la "esquiva" naturaleza sino al modo de establecer las relaciones espaciales, los cuales agudizan las condiciones de vulnerabilidad y amenaza.

Formar a los docentes y los estudiantes desde esta perspectiva no los coloca como meros objetos del espacio, sino como sujetos del propio y del global. Esta es la conjunción didáctica que se espera fomentar.

Con todo, desarrollar esta perspectiva requiere alta autonomía profesional, ingentes capacidades teóricas y metodológicas por parte de los profesores, además de unas buenas bases pedagógicas y geográficas, por cierto, con grados crecientes de trabajo cooperativo y en redes. Solo así será posible afrontar el currículo disgregado y sin consistencia teórica, la presión por la homogenización y la rendición de cuentas solo basada en el rendimiento de pruebas externas y estandarizadas en un contexto de mercantilización del hecho educativo.

\section{El caso Colombia}

La geografía escolar se ubica en el área de Ciencias Sociales, donde comparte la estructura curricular con historia, la constitución política y la democracia, según lo establecido en la Ley General de Educación o Ley 115 de 1994. Esta, en el artículo 23, define las áreas obligatorias y fundamentales que comprenden un mínimo del $80 \%$ del plan de estudios para la educación básica; allí, además de las Ciencias Sociales, están las áreas de: Ciencias naturales y Educación Ambiental; Educación Artística; Educación Ética y en Valores Humanos; Educación Física, Recreación y Deportes; Educación Religiosa; Humanidades, Lengua Castellana e Idiomas Extranjeros; Matemáticas y Tecnología e Informática. Para la educación media, la geografía no se considera propiamente, pero sí se ofrecen las Ciencias Políticas y Ciencias Económicas.

Esta estructura del currículo oficial colombiano, por áreas de enseñanza, abre el abanico de las disciplinas que se enseñan en cada una, y hace un llamado a la complementariedad de los contenidos y al diálogo de saberes, situación que aún genera incertidumbre y cierto escepticismo entre los docentes frente a la propuesta, puesto que muchos de ellos han sido formados desde la singularidad de las disciplinas y, más específicamente, desde contenidos propios de cada una de ellas. Por ello, esta concepción trae como consecuencia el reto de modernizar los procesos de enseñanza, es decir, la necesidad de incluir diversos métodos, medios y formas que dinamicen el aprendizaje, puesto que se rompe con la organización por asignaturas y se convoca al diálogo entre disciplinas.

La concepción de las ciencias sociales como área de enseñanza se ve ratificada, primero, con la expedición de los Lineamientos curriculares de ciencias sociales en el 2002, y luego con los Estándares básicos de competencias (2006); con estos documentos se fortalece la visión de las ciencias sociales como un conocimiento abierto, donde se reconocen diferentes objetos de estudio de los cuales las ciencias sociales se ocupan, generando diversas teorías y métodos que se estructuran desde disciplinas científicas que tienen algo en común: la pregunta por problemas de índole social, asumidos ya sea desde lo histórico, lo político, lo geográfico, lo sociológico o antropológico, entre otras. Todas estas son disciplinas que desde su especificidad contribuyen a relativizar, fortalecer y ampliar el conocimiento científico sobre lo social.

El concepto de área de enseñanza implica pluralidad, confluencia de disciplinas afines por su objeto de conocimiento; en el caso de las ciencias sociales, hoy ven ampliado su panorama con disciplinas como la política, la economía, la sociología y la antropología, entre otras, generando la posibilidad de abrir las ciencias sociales, como lo señala Wallerstein (1999). Hoy es necesario analizar la conveniencia o no de mantener la división disciplinar entre las distintas ciencias sociales a la hora de organizar el conocimiento escolar; es ineludible reconocer la estructura conceptual de las disciplinas que conforman el área de enseñanza para establecer nexos entre ellas. Así mismo, es importante reconocer otras formas de conocimiento sobre lo social procedentes de las artes y de la literatura que deben considerarse a la hora de planificar el proceso docente del área.

Esta situación hace que se piense más en los objetivos de la educación como componente rector del proceso docente, es decir, tener como punto de partida la finalidad de la educación desde las ciencias sociales y no simplemente los contenidos a enseñar. Es ahí donde la educación geográfica emerge como un referente importante en la organización del currículo, que facilite la comprensión y la explicación de los problemas ambientales y sociales en el mundo presente, como lo indica Souto (2011), una educación que busque formar para el mundo de la vida, por eso debe preguntarse por los problemas de la vida cotidiana, del mundo real.

La educación geográfica como finalidad de la enseñanza es compartida con los objetivos de la enseñanza de las ciencias naturales (Pulgarín, 2011), como se observa en los Estándares básicos de competencias científicas del 2004, documento en el cual se hace un llamado a la multidisciplinariedad, puesto que los estándares tienen una característica especial: se conciben para el aprendizaje de las ciencias, en plural, dando lugar a las ciencias naturales y a las ciencias sociales; además, están dirigidos hacia la formación de una actitud científica en la escuela. Este 
documento enfatiza cómo la realidad se presenta de manera fragmentada o separada por disciplinas y saberes, pero que el mundo real es uno, es un conjunto de cosas naturales y sociales (Santos, 2000), donde priman las relaciones entre las personas y las cosas, y entre las cosas mediadas por las personas, la realidad se encuentra de manera integrada, holística y global, y la escuela, como instancia socializadora, debe presentarla, estudiarla e investigarla de esta misma manera.

Finalmente, el currículo colombiano, a través de los diferentes referentes aquí señalados, reafirma que la finalidad de la educación pretende: instruir en las ciencias, desarrollar habilidades en saber hacery actuar con el conocimiento obtenido, con miras a planear propuestas transformadoras de la realidad que vivimos. No es otra cosa que educar en competencias, entendidas, según Perronoud (2004), como la capacidad de movilizar varios recursos cognitivos para hacer frente a un tipo de situaciones.

Para el caso de Medellín, se ha optado por elegir como cuestión socialmente viva la "emergencia ambiental" como la que se dio en abril y mayo del 2016. Su abordaje didáctico implica entender que la contaminación atmosférica es una expresión de un problema socioespacial, es decir, la contaminación atmosférica y el riesgo que implica son consecuencias de una determinada relación, ser humano-naturaleza, que se ha dado por décadas en Medellín, especialmente por la falta de control a la contaminación que genera la industria, el excesivo número de automotores, y la geoforma del denominado Valle de Aburrá, que no favorece la circulación de los agentes contaminantes en algunas épocas del año. Se percibe claramente que esta cuestión debe ser explicada de manera compleja, no solo desde la geografía ni desde las ciencias naturales, sino como un problema socioespacial en cuya explicación convergen ámbitos conceptuales de diversa procedencia. En ese sentido, se parte de una cuestión socialmente relevante, la contaminación atmosférica de Medellín, que implica un riesgo (ambiental para la salud y para la calidad de vida urbana), y se aborda didácticamente como un problema socioespacial (relación ser humano-naturaleza desde coordenadas espacio-temporales).

La contaminación atmosférica en Medellín como cuestión socialmente viva permite abordar ámbitos conceptuales en las relaciones sociedad-naturaleza, como: los riesgos ambientales y su relación con el desarrollo de las ciudades; la crisis ambiental como un fenómeno local-global; la experiencia espacial de los sujetos y su relación con un desarrollo sustentable; la contaminación atmosférica; el cambio del clima; y conceptos como atmósfera, clima, riesgo ambiental, ciudad, lugar, globalización y escala, desarrollo sostenible, ciudadanía. Metodológicamente, se propone la cartografía social como un método y un medio, que permite el razonamiento inductivo-deductivo y comparativo, que contrasta lo individual para construir posicionamientos y aprendizajes colectivos. Se tienen tres etapas o momentos del trabajo propuesto en el curso: premapeo, mapeo y posmapeo.
Desde esta perspectiva, la enseñanza y el aprendizaje de la geografía, tienen una lógica crítico-constructivista, la cual aprovecha el conocimiento derivado de la experiencia espacial del sujeto como insumo para la problematización y el aprendizaje de lo geográfico, mediante la interpelación que lleva a la movilización

\section{A modo de conclusión: lineamientos para la continuidad}

El curso B-learning que permitió al grupo orientador explicitar este lugar de enunciación está en un momento de diseño y a punto de comenzar el proceso formativo (julio del 2016). Su diseño considera elemento clave y diferenciador el hecho de no partir del currículo sino de los casos locales que configuran un determinado riesgo socioambiental, con el fin de darles un tratamiento didáctico que los comprenda como problemas socioespaciales, es decir, que no parta de los conceptos que se deben enseñar ni de las habilidades o competencias que deben adquirir los estudiantes, sino de la realidad situada y concreta.

En tal sentido, se han diseñado cuatro casos locales con el fin de que cada docente participante del curso estudie y analice por lo menos dos casos, el local y otro de una ciudad diferente a la que habita. El proceso permitirá que los participantes trasciendan la necesidad de establecer una relación unidimensional entre problema local y currículo nacional, y retomen elementos de los otros casos para incorporarlos en su ejercicio didáctico.

En términos del proceso del curso, en la primera parte los docentes participantes analizan los dos casos y en la segunda parte diseñan una secuencia didáctica, que, por supuesto, estará relacionada con su respectivo currículo, pero de una manera crítica y propositiva, fruto del enfoque socioespacial (relacional de lo universal-particular). Luego, los docentes realizarán su ejercicio práctico en el aula y se terminará con la valoración de la experiencia.

Este enfoque implica que el curso posibilite y valore como elemento formativo el diálogo y el intercambio de experiencias entre los docentes, por eso las estrategias del componente tanto presencial como virtual están diseñadas para tal fin. Al final, más que valorar la implementación de la secuencia didáctica en el aula, se va a evaluar el lugar de enunciación que tienen los docentes frente a la educación geográfica.

En ese sentido, más que permitir el desarrollo de las secuencias, lo que se espera identificar es si los docentes participantes entienden las potencialidades de darle un tratamiento socioespacial a un problema de riesgo ambiental (a una cuestión socialmente viva) y si se han posicionado en ese lugar de enunciación, es decir, en qué sentido los interpeló el enfoque propuesto. 
Se podrá verificar dicho posicionamiento ante la didáctica de la geografía, en cuanto vean con claridad posibilidades de superar la vieja dicotomía en la que se enfrascan algunos estudiosos del medio ambiente y las relaciones entre ser humano y ambiente, que ponen en un lado los enfoques deterministas y en el otro los enfoques construccionistas. Este enfoque no es dicotómico sino relacional. También se podrá observar si los docentes valoran la perspectiva constitutiva del espacio, que en lugar de fragmentar los problemas según campo de conocimiento y por conceptos, se posiciona en una perspectiva más cercana a las tramas o ámbitos conceptuales, en la que observa positivamente las matrices conceptuales de la geografía, incluso el debate del posibilismo, el determinismo ambiental y la geografía ambiental, y lo pone en diálogo con discursos disciplinares de la historia, la sociología, la antropología, la economía y otros más recientes como la ecología política, los estudios del territorio y la formación ciudadana.
En términos didácticos, lo que se debe analizar es si los docentes participantes ven que la perspectiva constitutiva y relacional del espacio les permite abordar cuestiones socialmente vivas, relevantes para el sujeto, es decir, parten del problema (o de la relación ser humano-naturaleza) y no del currículo, ni, mucho menos, del libro de texto. Observar esta posibilidad es ver que el sujeto no se percibe ni se entiende fuera del espacio sino en relación con este, lo cual permite resignificar algunas de las preguntas primigenias de la geografía que se ocupa de las relaciones ser humano-naturaleza, como: ¿existe un orden o un propósito en la naturaleza, en su funcionamiento?, ¿cómo incide la naturaleza los rasgos humanos (de personalidad, físicos, mentales)?, ¿cuál es el papel del ser humano en la modificación y la construcción de una segunda naturaleza?, entre otras.

\section{Referencias}

Bauman, Z. (2007). Los retos de la educación en la modernidad líquida. Barcelona: Gedisa Editores.

Ley 115 de 1994 (8 de febrero). Por la cual se expide la ley general de educación. Diario Oficial n.o 41.214.

Ministerio de Educación Nacional (2002). Lineamientos curriculares de ciencias sociales. Bogotá: Casa Editorial Ltda. del Ministerio de Educación Nacional de la República de Colombia.

Ministerio de Educación Nacional (2004). Estándares básicos de competencias científicas. Bogotá: Casa Editorial Ltda. del Ministerio de Educación Nacional de la República de Colombia.

Ministerio de Educación Nacional (2006). Estándares básicos de competencias. Bogotá: Casa Editorial Ltda. del Ministerio de Educación Nacional de la República de Colombia.

Perronoud, P. (2004). Diez nuevas competencias para enseñar. México D.F.: Gráficas Monte Albán.

Pulgarín, R. (2011). El espacio geográfico como objeto de enseñanza en el área de ciencias sociales. Recuperado de: www.sogeocol.edu.co

Santos, M. (2000). Naturaleza del espacio. En Técnica y tiempo. Razón y emoción. Barcelona: Editorial Ariel.

Souto González, X. M. (2011). Una educación geográfica para el siglo XXI: aprender competencias para ser ciudadano en el mundo global. Revista Virtual Anekumene, (1).

Wallerstein, I. (1999). Impensar las ciencias sociales. S. Guardado (trad.) (2a ed.). México, D.F.: Siglo XXI Editores. 